\title{
Evaluación de diferentes niveles de polidextrosa y azúcar en la elaboración de una jalea de guanábana (Annona muricata L.)
}

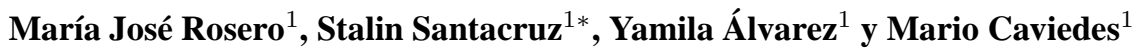 \\ ${ }^{1}$ Universidad San Francisco de Quito, Diego de Robles y Vía Interoceánica, \\ Quito, Ecuador. \\ *Autor principal/Corresponding author, e-mail: stalin.santacruz@gmail.com \\ Editado por/Edited by: Cesar Zambrano, Ph.D. \\ Recibido/Received: 17/12/2012. Aceptado/Accepted: 27/04/2013. \\ Publicado en línea/Published on Web: 28/06/2013. Impreso/Printed: 06/06/2013.
}

\begin{abstract}
The task of the present research was to quantify the effect of polydextrose and sugar contents on the manufacture of guanabana jam. The sugar content was, 50, 55 and $60 \mathrm{~g}$, whereas for polydextrose was $0,0.30,0.60$ and $0.90 \mathrm{~g}$. In each case the contents were based on $100 \mathrm{~g}$ of product. A completely randomized design with $3 \times 4$ factorial arrangement was used. The 12 treatments of the design were evaluated by using gel firmness, Brix and $\mathrm{pH}$. Three treatments were chosen for the sensory analysis and the results showed that the best jelly was the one containing $60 \%$ sugar and $0.90 \%$ polydextrose.
\end{abstract}

Keywords. jam, polydextrose, Annona muricata

\section{Resumen}

El objetivo de esta investigación fue el de cuantificar la respuesta de diferentes niveles de polidextrosa y azúcar para la formulación adecuada de una jalea de guanábana; para lo cual, se evaluaron diferentes formulaciones con tres niveles de azúcar: 50, 55 y 60 g y cuatro niveles de polidextrosa: $0.0,0.30,0.60$ y $0.90 \mathrm{~g}$. En cada caso los niveles fueron basados en $100 \mathrm{~g}$ de producto. En la fase experimental se utilizó un diseño experimental completamente al azar (DCA) con arreglo factorial de $3 \times 4$. Se evaluaron las respuestas de los 12 tratamientos de las variables penetrabilidad (dureza del gel), grados brix y potencial de hidrógeno $(\mathrm{pH})$. Se seleccionaron tres para la evaluación sensorial. Los resultados permitieron demostrar que el prototipo más aceptado fue el que contenía $60 \mathrm{~g}$ de azúcar y 0.90 g de polidextrosa, constituyéndose en la formulación final.

Palabras Clave. guanábana, jalea, polidextrosa, Annona muricata

\section{Introducción}

Las annonas comprenden un grupo de más de 50 especies de arbustos y árboles pequeños de los cuales 8 a 10 producen frutos comestibles [1]. El sabor de la mayoría de las annonas es dulce, cremoso y agradable. La guanábana Annona muricata L. pertenece a la familia Anonácea a la que también pertenecen el anón, el mamón, y la chirimoya [2]. La guanábana es originaria de América tropical, es una fruta climatérica, muy susceptible al frío, y prospera bien en tierras húmedas tropicales por debajo de los 1000 metros sobre el nivel del mar. Su pulpa contiene carbohidratos y varios minerales como fósforo, hierro, sodio y vitaminas como A, B, C y niacina [2]. La fruta se utiliza para hacer bebidas refrescantes y dar sabor a pastas y helados, con la pulpa se puede hacer jaleas y conservas [3].

La polidextrosa se conoce con el nombre comercial de Litesse II o polidextrosa super mejorada FCC, es un polímero condensado con uniones aleatorias de polímeros de D-glucosa con algunos enlaces de sorbitol y ácido cítrico; es altamente soluble en agua pero parcialmente soluble en solventes orgánicos [4]. La polidextrosa actúa como fibra soluble en el organismo humano y es resistente a la hidrólisis de las enzimas digestivas por lo tanto, provee de varios beneficios a la salud. [5].

La pectina es una agente gelificante natural presente en las frutas; actúa en la gelificación de mermeladas y jaleas. La molécula de pectina está esencialmente compuesta por largas cadenas de ácido poligalacturónico parcialmente metoxilado. Asimismo, la pectina aumenta su disponibilidad a medida que la fruta madura; la adición de azúcar tiene un efecto deshidratante sobre la pectina y reduce su solubilidad. El edulcorante más utilizado en la elaboración de jaleas es la sacarosa, o azúcar blanca [6].

La evaluación sensorial de alimentos o de otros produc- 


\begin{tabular}{clccc}
\hline Variable & \multicolumn{1}{c}{$\begin{array}{c}\text { Fuente de } \\
\text { variación }\end{array}$} & GL & CM & Fc \\
\hline Penetrabilidad & Total & 35 & - & - \\
& Tratamiento & 11 & 674.21 & $146.25^{*}$ \\
& Azúcar (A) & 2 & 85.75 & $18.60^{*}$ \\
& Polidextrosa (B) & 3 & 1874.26 & $405.56^{*}$ \\
& Interacción AxB & 6 & 270.34 & $58.64^{*}$ \\
& Error experimental & 24 & 4.61 & \\
\hline Grados Brix & Total & 35 & - & - \\
& Tratamiento & 11 & 1.78 & $8.90^{*}$ \\
& Azúcar (A) & 2 & 2.67 & $13.35^{*}$ \\
& Polidextrosa (B) & 3 & 0.12 & $0.60 \mathrm{NS}^{*}$ \\
& Interacción AxB & 6 & 2.32 & $11.60^{*}$ \\
& Error experimental & 24 & 0.20 & \\
\hline Potencial de & Total & 35 & - & - \\
Hidrógeno & Tratamiento & 11 & 0.02154 & $37.72^{*}$ \\
& Azúcar (A) & 2 & 0.0070 & $12.26^{*}$ \\
& Polidextrosa (B) & 3 & 0.0440 & $77.05^{*}$ \\
& Interacción AxB & 6 & 0.01516 & $26.54^{*}$ \\
& Error experimental & 24 & 0.00057 & \\
\hline$*$ Significativo p $\leq 0.05$ & NS No significativo p $\leq 0.05$ \\
\hline
\end{tabular}

Tabla 1: Resumen del análisis de varianza (ANOVA) de las variables penetrabilidad (dureza del gel), grados brix y potencial de hidrógeno (pH) de los doce tratamientos

tos puede ser usada para realizar un análisis preliminar del sabor del alimento y proveer indicios para luego realizar análisis físico químicos. Asimismo, cambios en las características sensoriales pueden proveer información sobre cambios en las características físicas de un alimento [7]

El objetivo del presente estudio fue el de cuantificar la respuesta de diferentes niveles de polidextrosa y azúcar para la formulación adecuada de una jalea de guanábana.

\section{Materiales y Métodos}

\section{Producto}

Para la elaboración del la formulación de la jalea de guanábana se utilizarán diferentes proporciones de ingredientes por cada $100 \mathrm{~g}$. La fórmula inicial contuvo zumo de guanábana, azúcar blanca, pectina de alta metoxilación, ácido cítrico y benzoato de sodio, obtenidos en el mercado local. La polidextrosa "Litesse" fue adquirida de Danisco.

\section{Características del producto}

Para la evaluación de las características de la formulación se realizó un experimento preliminar de penetrabilidad para determinar la dureza del gel [8]. La penetrabilidad del producto inicial fue de $210(1 / 10 \mathrm{~mm})$ que al compararla con la penetrabilidad de $193(1 / 10$ $\mathrm{mm}$ ) de la jalea de mora "Snob", permitió obtener una menor dureza del gel. El producto final fue desarrollada considerando diferentes porcentajes de polidextrosa y azúcar por cada $100 \mathrm{~g}$ de producto generando doce tratamientos que fueron la combinación de cuatro niveles de polidextrosa "Litesse" $(0.00 ; 0.30 ; 0.60 ; 0.90 \mathrm{~g})$ y tres niveles de azúcar $(50.00 ; 55.00$ y $60.00 \mathrm{~g})$.

\section{Fase experimental y análisis estadístico}

Los 12 tratamientos fueron evaluados bajo un diseño experimental completamente al azar (DCA) con arreglo factorial de $3 \times 4$. Se cuantificaron tres variables: penetrabilidad (dureza del gel), grados brix y $\mathrm{pH}$; utilizando diferentes métodos y normas estandarizadas. Para dureza del gel el método del penetración [8], grados brix, norma INEN 380 [9] y pH norma INEN 389 [10].

Para la evaluación de los resultados se utilizó un análisis de varianza de una vía (ANOVA) con arreglo factorial para cada variable bajo estudio y una prueba de separación de medias de "Tukey" para determinar la significación estadística de las diferencias entre las medias y establecer sus rangos [11]. Para la selección de los mejores tratamientos considerando las tres variables, se utilizó valores de ponderación en relación con el nivel de importancia que se le asignó a cada variable en el siguiente orden: dureza del gel, grados brix y $\mathrm{pH}$.

\section{Evaluación sensorial}

Para el análisis y estudio de aceptabilidad del producto, se aplicaron diferentes métodos estadísticos: análisis de varianza de dos vías [11], pruebas utilizando el modelo Thurstoniano (Norma ASTM E2262 - 03) e índice $\mathrm{R}[12,13]$. Asimismo, se utilizó la metodología de la AOAC 1990 para el análisis físico-químico del producto.

\section{Resultados y discusión}

\section{Análisis de varianza}

En la tabla 1 se presentan las ANOVAS para las variables penetrabilidad, grados brix y potencial hidrógeno; 


\begin{tabular}{|c|c|c|c|c|c|}
\hline \multicolumn{3}{|c|}{ Tratamientos } & \multicolumn{3}{|c|}{ Variables } \\
\hline \multirow{2}{*}{$\mathrm{N}^{\circ}$} & \multicolumn{2}{|c|}{ Niveles } & \multirow{2}{*}{ Penetrabilidad ${ }^{*}$} & \multirow{2}{*}{ Grados Brix* } & \multirow[t]{2}{*}{$\mathrm{pH}^{*}$} \\
\hline & Azúcar (g) & Polidextrosa $(\mathrm{g})$ & & & \\
\hline 12 & 60.00 & 0.90 & $187.0 \mathrm{a}$ & $65.37 \mathrm{~cd}$ & $3.22 \mathrm{abc}$ \\
\hline 4 & 50.00 & 0.90 & $186.7 \mathrm{a}$ & $65.53 \mathrm{~cd}$ & $3.25 \mathrm{a}$ \\
\hline 8 & 55.00 & 0.90 & $183.7 \mathrm{a}$ & $66.40 \mathrm{abc}$ & $3.25 \mathrm{a}$ \\
\hline 11 & 60.00 & 0.60 & $177.3 \mathrm{~b}$ & $65.27 \mathrm{~cd}$ & $3.17 \mathrm{bc}$ \\
\hline 6 & 55.00 & 0.30 & $175.3 \mathrm{~b}$ & $65.37 \mathrm{~cd}$ & $3.21 \mathrm{abc}$ \\
\hline 2 & 50.00 & 0.30 & $164.3 \mathrm{c}$ & $65.20 \mathrm{~cd}$ & $3.15 \mathrm{~cd}$ \\
\hline 10 & 60.00 & 0.30 & $157.0 \mathrm{~d}$ & $66.67 \mathrm{abc}$ & $3.18 \mathrm{abc}$ \\
\hline 9 & 60.00 & 0.00 & $155.7 \mathrm{~d}$ & $67.27 \mathrm{a}$ & $3.16 \mathrm{bc}$ \\
\hline 7 & 55.00 & 0.60 & $154.7 \mathrm{~d}$ & $67.00 \mathrm{ab}$ & $3.13 \mathrm{~d}$ \\
\hline 3 & 50.00 & 0.60 & $154.3 \mathrm{~d}$ & $65.53 \mathrm{~cd}$ & $3.15 \mathrm{~cd}$ \\
\hline 1 & 50.00 & 0.00 & $153.7 \mathrm{~d}$ & $64.92 \mathrm{~d}$ & $3.08 \mathrm{~d}$ \\
\hline 5 & 55.00 & 0.00 & $144.3 \mathrm{e}$ & $65.67 \mathrm{bcd}$ & $3.23 \mathrm{ab}$ \\
\hline
\end{tabular}

Tabla 2: Resumen de la prueba de significación de medias (Tukey) de las variables penetrabilidad (dureza del gel), grados brix y potencial de hidrógeno $(\mathrm{pH})$ de los doce tratamientos

de estos se desprende que para la variable penetrabilidad se encontraron diferencias significativas $(p<0.05)$ para tratamientos: contenidos de azúcar (A), contenido polidextrosa (B) e interacción de los contenidos de azúcar y polidextrosa (AxB). Estos resultados demuestran que hubo efectos positivos de los diferentes contenidos de azúcar y polidextrosa en los tratamientos estudiados en relación con la dureza del gel.

Por otra parte, para grados brix se encontraron efectos significativos $(\mathrm{p}<0.05)$ para el factor principal azúcar y no significativos para polidextrosa; asimismo, la interacción de los niveles de azúcar y polidextrosa fue estadísticamente significativa. Estas respuestas para la variable grados brix, mostraron que la utilización de diferentes niveles de polidextrosa no presentaron una respuesta positiva en la cuantificación de esta variable al considerar su efecto individual.

Del análisis de la varianza (ANOVA) para el $\mathrm{pH}$ se encontraron diferencias estadísticas $(\mathrm{p}<0.05)$ tanto para los efectos principales azúcar y polidextrosa como para la interacción de azúcar y polidextrosa, lo que es un indicativo de que el $\mathrm{pH}$ de los diferentes tratamientos bajo estudio mostró marcadas variaciones.

Los resultados significativos en las tres variables bajo estudio para la interacción entre los niveles de azúcar y de polidextrosa, nos indican que los factores no son independientes, sin más bien que actúan conjuntamente y que esta interacción implica que las diferencias de las respuestas en el factor B polidextrosa, varían de acuerdo al nivel de azúcar, factor $\mathrm{A}$.

\section{Pruebas de significación}

En la tabla 2 se muestran las pruebas de separación de medias de Tukey para las tres variables cuantificadas de respuesta. Se encontraron diferencias estadísticas significativas para penetrabilidad; presentando mayores magnitudes para esta variable los tratamientos 12,4 y 8 siendo iguales estadísticamente y ocupando el mismo rango de significación. Al comparar estos valores con los de penetrabilidad de la jalea de mora marca "Snob" 193 $(1 / 10 \mathrm{~mm})$ se observa que son en magnitud similares a los de la jalea comercial.

Para la variable grados brix, los resultados según la prueba de Tukey permitieron diferenciar a los tratamientos, mostrando las combinaciones 9,7 y 10 valores superiores y al compararla con el parámetro establecido en la Norma CODEX STAM 296-2009 para confituras, jaleas y mermeladas que establece un rango de "60 a $65 \%$ o superior", estos tres tratamientos mostraron valores superiores al $65 \%$ y estadísticamente ocuparon el mismo rango de significación.

De acuerdo con la prueba de Tukey para la variable $\mathrm{pH}$, los tratamientos 8, 4 y 5 mostraron los valores más altos, sin embargo, todos los tratamientos estuvieron dentro del rango de $\mathrm{pH}$ establecido con un mínimo de 2.8 y un máximo de 3.5 [6].

\section{Evaluación sensorial y estudio de aceptabilidad del producto}

Con base a los valores de ponderación asignados a las variables penetrabilidad [3]; grados brix [2] y pH [1] se seleccionaron los tratamientos 8 (55 g de azúcar / 0.90 polidextrosa), 12 (60 g de azúcar / 0.90 polidextrosa) y 4 (50 g de azúcar / 0.90 polidextrosa). Los cuales fueron considerados para la etapa de evaluación sensorial y estudio de aceptabilidad del producto. La codificación de las muestras se la realizó mediante el programa "Desing Express"; la muestra A, tratamiento 4, se codificó como (394), la B, tratamiento 8, como (170), y la C, tratamiento 12 como (622). En general las respuestas fueron similares para el nivel de agrado de las tres muestras de la jalea de guanábana expresada por los 100 consumidores. Los resultados se concentraron mayormente en las categorías de 6 a 9 de la escala hedónica, lo que es un indicativo de que las tres muestras tuvieron aceptación en los consumidores; sin embargo, la muestra 622 (C) tuvo el mayor nivel de agrado. 


\begin{tabular}{lccc}
\hline FV & GL & CM & Fc \\
Total & 299 & - & - \\
Jueces & 993.272 & $2.56^{*}$ & \\
Tratamientos & 2 & 2.425 & $1.89 \mathrm{NS}$ \\
Error experimental & 198 & 1.278 & \\
\hline${ }^{*}$ Significativo $\mathrm{p}<0.05$ & NS No significativo $\mathrm{p}<0.05$
\end{tabular}

Tabla 3: Resumen del análisis de varianza (ANOVA) del nivel de aceptación de tres tratamientos seleccionados

\section{ANOVA de dos vías}

En la tabla 3 se presenta el ANOVA de dos vías de la prueba del nivel de aceptación de los tres tratamientos (muestras). Se encontraron diferencias estadísticas $(p<0.05)$ para jueces pero no para tratamientos, es decir que las tres muestras presentaron un nivel de agrado similar para los consumidores.

\section{Modelo Thurstoriano}

La tabla 4 presenta los valores d' y los límites máximo y mínimo obtenidos a partir de pares de muestras para la escala hedónica de 9 puntos de acuerdo con el modelo Thurstoniano.

Los jueces expresaron un nivel de agrado similar tanto para la muestra $\mathrm{B}$ como para la $\mathrm{C}$, por esta razón fue el par de muestras con menor dispersión de valores. Asimismo, la mayor dispersión se encontró entre A y C y una dispersión intermedia entre A y B. En base a este análisis, los jueces expresaron un nivel de agrado similar para los pares de muestras A y B y B y C; con un nivel menor de agrado para el par A y $\mathrm{C}$.

\section{Índice $\mathbf{R}$}

Los valores de índice $\mathrm{R}$ para los pares de muestras se presentan en la tabla 5 .

Los resultados del índice $\mathrm{R}$ determinarón que los muestras 394 (A) y 170 (B) fueron estadísticamente iguales $\mathrm{y}$ por lo tanto, los jueces expresaron un nivel de agrado similar; por otro lado, las muestras 394 (A) y 622 (C) presentaron diferencias estadísticas, por lo que los jueces expresaron diferentes niveles de agrado tanto para A como para C. Finalmente, la comparación entre las muestras 170 (B) y 622 (C) fueron estadísticamente iguales, y se encontraron en el límite de los valores, del nivel de agrado de la estimación de los jueces. Adicionalmente, se observó que un $67 \%$ de jueces indicó el mismo grado de preferencia para dos muestras, y solo un $33 \%$ de estos estableció una diferencia entre las tres muestras en el nivel de agrado.

Los resultados encontrados utilizando los tres modelos estadísticos permitieron determinar que según el ANO-

\begin{tabular}{ccccc}
\hline $\begin{array}{c}\text { Pares de } \\
\text { muestras }\end{array}$ & d' & sd' & $\begin{array}{c}\text { Límite } \\
\text { máximo }\end{array}$ & $\begin{array}{c}\text { Límite } \\
\text { mínimo }\end{array}$ \\
\hline 394 (A) con 170 (B) & 0.203 & 0.18748 & 0.57047 & -0.16447 \\
394 (A) con 622 (C) & 0.367 & 0.19191 & 0.74315 & -0.000915 \\
170 (B) con 622 (C) & 0.163 & 0.19560 & 0.54638 & -0.22038 \\
\hline
\end{tabular}

Tabla 4: Comparaciones entre pares de muestras utilizando el modelo Thurstoniano para categorías de una escala de 9 puntos (ASTM E2262-03)

\begin{tabular}{ccc}
\hline $\begin{array}{c}\text { Pares de } \\
\text { muestras }\end{array}$ & $\begin{array}{c}\text { IR Calculado } \\
(\%)\end{array}$ & $\begin{array}{c}\text { IR tabulado + } \\
50 \%\end{array}$ \\
\hline $\mathrm{AB}$ & $47.71 \mathrm{NS}$ & 57.89 \\
$\mathrm{AC}$ & $58.80^{*}$ & 57.89 \\
$\mathrm{BC}$ & $57.82 \mathrm{NS}$ & 57.89 \\
\hline
\end{tabular}

${ }^{*}$ Significativo $\mathrm{p}<0.05$

NS No significativo $\mathrm{p}<0.05$

Tabla 5: Índice $\mathbf{R}$ para pares de muestras [7]

VA no hubo diferencias estadísticas para el nivel de agrado entre los tres tratamientos; por otro lado, el modelo Thurstoniano indicó que la menor dispersión de valores se encontró al relacionar las muestras 170 (B) con 622 (C). Asimismo, el índice $\mathrm{R}$ determinó diferencias estadísticas entre las muestras 394 (A) y 622 (C). Considerando los tres modelos estadísticos evaluados se seleccionó la muestra 622 (C) con $60 \mathrm{~g}$ de azúcar y $0.90 \mathrm{~g}$ de polidextrosa, la que mostró una mayor tendencia de aceptación por parte de los consumidores, por lo cual fue la formulación final del producto para la futura producción industrial.

\section{Conclusiones}

Los resultados de este estudio determinaron la posibilidad de obtener una jalea de guanábana utilizando como fuente de fibra soluble a la polidextrosa, seleccionándose a la formulación que contenía $60 \mathrm{~g}$ de azúcar y $0.90 \mathrm{~g}$ de polidextrosa por cada $100 \mathrm{~g}$ de producto. El producto final evaluado sensorialmente fue de agrado de los consumidores obteniendo un promedio de 7 en la escala hedónica de 1 al 9. Se recomienda realizar análisis cuantitativos de polidextrosa de acuerdo con la norma oficial AOAC 2000.11.

\section{Agradecimientos}

Los autores del estudio agradecen la colaboración brindada por los asistentes del laboratorio de análisis de alimentos y de la planta piloto del Departamento de Ingeniería de Alimentos de la USFQ para los procesos de elaboración del producto y en la cuantificación de las variables del producto final.

\section{Referencias}

[1] Bailey, M. 1928. "Cultivo de plantas tropicales". Toloma Colombia Cyclopedia.

[2] Arango, T. 1975. "La guanábana". Revista Esso Agrícola, 21(2).

[3] Avilán, L.; Leal, F.; Bautista, D. 1992. "Manual de fruticultura". Segunda edición Tomo I. Venezuela: Ed. América.

[4] 296, C. S. 2009. "Norma del codex para las confituras, jaleas y mermeladas".

[5] Craig, F.; Holden, J.; Troup, M.; Averbach, H.; Frier, I. 1998. "Polidextrose as soluble fiber: Physiological and analytical aspects". Publication no. W-1998-0427-03 F. American Association of Cereal Chemists, 43(5). 
[6] Arthey, D.; Ashurst, P. 1997. "Procesado de Frutas". Zaragoza: Ed. Acribia.

[7] O’Mahony, M. 1986. "Sensory evaluation of food. Statistical methods and procedures". Copyright by Marcel Dekne Inc.

[8] Ott, D. 1992. "Manual de laboratorio de ciencia de los alimentos". Zaragoza Ed. Acribia.

[9] INEN-389. 1985. "Conservas Vegetales: Determinación de la Concentración del ion hidrógeno $(\mathrm{pH})$ ". Instituto Ecuatoriano de Normalización. Primera revisión. Ecuador.

[10] INEN-380. 1985. "Conservas Vegetales: Determinación de Sólidos Solubles. Método Refrectométrico". Instituto Ecuatoriano de Normalización. Primera revisión. Ecuador.

[11] Sanchez, J. 2011. "Introducción al diseño experimental". Quito, Ecuador.

[12] ASTM. 2003. "Standard Practice for Estimating Thurstinian Discriminal Distances". International Designation: E 2262 Ü 03. United States.

[13] Bi, J. 2006. "Statistical Analyses for R - Index". Journal of Sensory Studies. 